\title{
Starspot activity and superflares on solar-type
}

\section{stars}

\author{
Hiroyuki Maehara ${ }^{1}$, Yuta Notsu, ${ }^{2}$, Shota Notsu ${ }^{2}$, Kosuke Namekata $^{2}$, \\ Satoshi HoNDA ${ }^{3}$, Takako T. ISHII ${ }^{4}$, Daisaku NoGAMI ${ }^{2}$ and Kazunari \\ SHIBATA ${ }^{4}$ \\ ${ }^{1}$ Okayama Astrophysical Observatory, National Astronomical Observatory of Japan, NINS \\ ${ }^{2}$ Department of Astronomy, Graduate School of Science, Kyoto University \\ ${ }^{3}$ Nishi-Harima Astronomical Observatory, Center for Astronomy, University of Hyogo \\ ${ }^{4}$ Kwasan and Hida Observatories, Graduate School of Science, Kyoto University \\ *E-mail: h.maehara@oao.nao.ac.jp \\ Received ; Accepted
}

\begin{abstract}
We analyze the correlation between starspots and superflares on solar-type stars using observations from the Kepler mission. The analysis shows that the observed fraction of stars with superflares decreases as the rotation period increases and as the amplitude of photometric variability associated with rotation decreases. We found that the fraction of stars with superflares among the stars showing large-amplitude rotational variations, which are thought to be the signature of the large starspots, also decreases as the rotation period increases. The small fraction of superflare stars among the stars with large starspots in the longer-period regime suggests that some of the stars with large starspots show a much lower flare activity than the superflare stars with the same spot area. Assuming simple relations between spot area and lifetime and between spot temperature and photospheric temperature, we compared the size distribution of large starspot groups on slowly-rotating solar-type stars with that of sunspot groups. The size distribution of starspots shows the power-law distribution and the size distribution of larger sunspots lies on this power-law line. We also found that frequency-energy distributions for flares originating from spots with different sizes are the same for solar-type stars with superflares and the Sun. These results suggest that the magnetic activity we ob-
\end{abstract}


serve on solar-type stars with superflares and that on the Sun is caused by the same physical processes.

Key words: stars: activity — stars: flare — stars: solar-type — starspots

\section{Introduction}

Solar flares are sudden energy releases in the solar atmosphere caused by the magnetic reconnection (e.g., Shibata \& Magara 2011). The typical energy released by solar flares ranges from $10^{28}$ to $10^{32}$ erg. Similar flares and larger "superflares" have been observed on various types of stars including solar-type stars (G- and late F-type main sequence stars; e.g., Weaver \& Naftilan 1973; Schaefer et al. 2000). The recent space-based observations revealed that not only rapidly rotating and young solartype stars but also slowly-rotating stars like our Sun exhibit superflares with the bolometric energy of $10^{33}$ to $10^{35} \mathrm{erg}$, which are 10-1000 times larger than the energy of the largest solar flares. (e.g., Maehara et al. 2012; Shibayama et al. 2013; Maehara et al. 2015).

Most of superflare stars show quasi-periodic light variations with the period and amplitude of $\sim 1$-30 days and $\sim 0.1-10 \%$. By analogy with the time variations in the total solar irradiance due to sunspots (e.g., Kopp et al. 2005), the brightness variations of superflare stars are thought to be caused by the rotation of stars and the superflare stars have much larger starspots than our Sun (e.g., Notsu et al. 2013). According to Shibata et al. (2013), the energy released by superflares are basically a part of magnetic energy stored around starspots if we assumed that the magnetic energy can be estimated from the area of starspots and the starspot area corresponds to the amplitude of light variations.

High-dispersion spectroscopy of superflare stars (Notsu et al. 2015a; Notsu et al. 2015b) revealed that projected rotation velocity $v \sin i$ of superflare stars is consistent with the rotation velocity estimated from the period of light variations. Notsu et al. (2015b) found that there is a clear correlation between the intensity of the chromospheric Ca II $8542 \AA$ line and the amplitude of quasi-periodic light variations of superflare stars. These results suggest that the photometric variations of superflare stars are caused by the rotation of the star and the amplitude of brightness variations is a good indicator of the chromospheric activity. Moreover, Karoff et al. (2016) reported that superflare stars show higher intensities of the $\mathrm{Ca}$ II $\mathrm{H}$ and $\mathrm{K}$ lines than ordinary solar-type stars. This result indicates that superflare stars show higher magnetic activity than ordinary solar-type stars including our Sun. Since the existence of large starspots (up to $\sim 10 \%$ of stellar hemisphere) is thought to be a key factor to produce superflares, it is important to investigate the statistical properties of large starspots (e.g., pro- 
duction rate and size distribution of starspots) and their dependence on stellar properties (e.g., rotation period) for the understanding of superflares. In this paper, we report (1) the relation between the flare activity and properties of starspots on solar-type stars, (2) the production rate and size distribution of starspots on solar-type stars, and (3) the comparison between the starspot-superflare connection on solar-type stars and the sunspot-solar flare connection on the Sun.

\section{Data and method}

\subsection{Sample selection}

We selected solar-type stars (early G- and late F-type main sequence stars) from the Kepler data set by using the effective temperature $\left(T_{\text {eff }}\right)$ and the surface gravity $(\log g)$ of the star. The dynamo activity is thought to correlate with the stellar mass, which is related to the effective temperature, and rotation period of the star (e.g., Kippenhahn \& Weigert 1990). In order to compare the spot activity of the solar-type stars and that of the Sun, we select the solar-type stars with the temperature similar to the Sun. Here we used the selection criteria of $5600 \mathrm{~K}<T_{\text {eff }}<6300 \mathrm{~K}$ and $\log g>4.0$ for the selection of solar-type stars. We also selected the stars exhibiting superflares from the flare star list in Shibayama et al. (2013). In previous studies about superflares (Maehara et al. 2012; Shibayama et al. 2013; Candelaresi et al. 2014), we selected G-, K-, and M-dwarfs as the target of search for superflares by using stellar parameters in the original Kepler Input Catalog (Brown et al. 2011). Since the temperature and surface gravity of the stars taken from Brown et al. (2011) are slightly different from those in Huber et al. (2014) (e.g., systematic difference in effective temperature), some stars selected by using the above criteria are not included in the G-dwarf samples in Shibayama et al. (2013). In order to avoid mismatch of the data caused by the difference in stellar parameters between Brown et al. (2011) and Huber et al. (2014), we selected samples that fulfill the both criteria: (1) $5600 \mathrm{~K}<T_{\text {eff }}<6300 \mathrm{~K}$ and $\log g>4.0$ in Huber et al. (2014); (2) $5100 \mathrm{~K}<T_{\text {eff }}<6000 \mathrm{~K}$ and $\log g>4.0$ in Brown et al. (2011), which is the same criteria as those used in Shibayama et al. (2013). The total number of samples is 64,239. The typical length of observations to search for superflares is $\sim 480$ days (Shibayama et al. 2013).

\subsection{Bolometric energy of superflares}

We used the data set of superflares detected by Shibayama et al. (2013). However, since the effective temperature of the stars taken from Brown et al. (2011) is approximately $200 \mathrm{~K}$ higher than those from Huber et al. (2014), we recalculated the bolometric energy of each superflare by using the stellar parameters taken from Huber et al. (2014) and the Kepler PDC light curve (DR24). The bolometric 
energy emitted by each flare is estimated by time integration of the change in the stellar luminosity caused by the flare with the same manner as Shibayama et al. (2013).

\subsection{Rotation period and amplitude of light variations}

McQuillan et al. (2014) derived the rotation period and median amplitude of periodic variability of main-sequence stars with $T_{\text {eff }}<6500 \mathrm{~K}$. In our analysis, rotation period and amplitude of light variations of our samples are taken from McQuillan et al. (2014). The rotation periods and amplitudes of 9,383 stars among 64,239 solar-type samples were detected. The typical observation length for searching for the period and amplitude of light variations is $\sim 1400$ days (McQuillan et al. 2014).

\subsection{Area of starspots}

We estimated the area of starspots from the amplitude of brightness variations and radius of the stars. The normalized amplitude of rotational light variation of the $\operatorname{star}(\Delta F / F)$ with the apparent starspot $\operatorname{area}\left(A_{\text {spot }}^{*}\right)$ can be written as

$$
\frac{\Delta F}{F}=\frac{A_{\mathrm{spot}}^{*}\left(T_{\mathrm{star}}^{4}-T_{\mathrm{spot}}^{4}\right)}{A_{\mathrm{star}} T_{\mathrm{star}}^{4}},
$$

where $A_{\text {star }}$ indicates the apparent area of the star, $T_{\text {star }}$ and $T_{\text {spot }}$ are the temperature of unspotted photosphere of the star and starspot, respectively. The temperature difference between stellar photosphere and starspots depends on the photospheric temperature of the stars (e.g., Berdyugina 2005). We estimated the temperature difference between photosphere and spots $\left(T_{\text {star }}-T_{\text {spot }}\right)$ by using the following equation,

$$
\Delta T\left(T_{\text {star }}\right)=T_{\text {star }}-T_{\text {spot }}=3.58 \times 10^{-5} T_{\text {star }}^{2}+0.249 T_{\text {star }}-808,
$$

which was derived from the second order polynomial fit to the data for all stars except for EK Dra in table 5 of Berdyugina (2005). The RMS of residuals of the fit is $220 \mathrm{~K}$. Therefore, from equation (1) and (2), we can estimate the area of starspots normalized by the area of solar hemisphere $\left(A_{\text {spot }}\right)$ as follows:

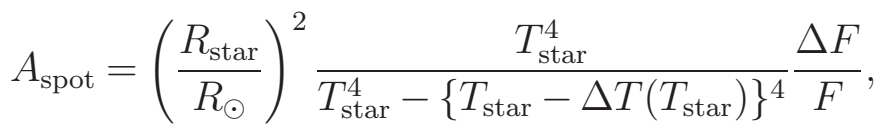

where $R_{\mathrm{star}} / R_{\odot}$ is stellar radius in units of the solar radius. The equations (1) and (3) are applicable only if (a) there are a few large spot groups on the surface of the star and (b) the lifetime of spots is much longer than the rotation period. As discussed in appendix 1, in the case of the solar-type stars showing large amplitude brightness variations, the typical number of major spot groups which contribute to the photometric light variations is 1-3. The typical lifetime of such spots ranges from 
$\sim 50$ days to $\sim 300$ days, which is longer than the rotation period of solar-type stars $(<40$ days). Therefore we can estimate the area of large starspots on the solar-type stars by using equation (3). In the later analysis, $R_{\mathrm{star}} / R_{\odot}$ and $T_{\text {star }}$ are taken from Huber et al. (2014) and $\Delta F / F$ are taken from McQuillan et al. (2014).

\section{Results}

Figure 1 (a) shows the scatter plot of the total spot-group area of solar-type stars in units of the area of solar hemisphere $\left(A_{1 / 2 \odot}=3 \times 10^{22} \mathrm{~cm}^{2}\right)$ as a function of the rotation period $\left(P_{\text {rot }}\right)$. Small dots indicate all solar-type stars $\left(5600<T_{\text {eff }}<6300 \mathrm{~K}, \log g>4.0\right)$ and open squares indicate solar-type stars showing superflares. Please note that there are many "inactive" stars whose area of starspots $<10^{-3} A_{1 / 2 \odot}$. Approximately $85 \%(54,856 / 64,239)$ stars are not plotted in figure 1 (a) since the amplitude of light variations is smaller than the detection limit. The largest area of starspots on solartype stars in a given period bin is roughly constant $\left(\sim 5 \times 10^{-2} A_{1 / 2} \odot\right)$ in the period range of $P_{\text {rot }}<12$ days. However, in the period range of $P_{\text {rot }}>12$ days, the largest starspot area decreases as the rotation period increases. The stars showing superflares tend to have shorter rotation period and larger starspot area. Figure 1 (b) and (c) show the observed fraction of the stars showing superflares as a function of the rotation period and area of starspots, respectively. As shown in figure 1 (b), the fraction of flare stars decreases as the rotation period increases in the range of the rotation period $P_{\text {rot }}>3$ days. In the period range of $P_{\text {rot }}<3$ days, the observed fraction of superflare stars is almost constant. The previous studies (e.g., Maehara et al. 2012) pointed out that the flare frequency as a function of the rotation period shows the similar decrease trend and "saturation" in the period range of $P_{\text {rot }}>3$ and $P_{\text {rot }}<3$ days. Moreover, the fraction of superflare stars also depends on the total area of starspots. Fraction of superflare stars rapidly decreases as the starspot area decreases as presented in figure 1 (c). In case of the stars whose area of starspots exceeds $10^{-1.5} A_{1 / 2} \odot$, the fraction of superflare stars is $\sim 8 \%$. The fraction decreases to $\sim 0.8 \%, \sim 0.5 \%$, and $\sim 0.02 \%$ as the spot area decreases to $10^{-2.0}-10^{-1.5} A_{1 / 2 \odot}, 10^{-2.5}-10^{-2.0} A_{1 / 2 \odot}$, and $10^{-3.0}-10^{-2.5} A_{1 / 2 \odot}$.

\subsection{Fraction of the stars with large starspots as a function of rotation period}

Figure 2 presents the ratio of the number of stars with a given starspots area to the total number of stars with the starspot area $>10^{-3} A_{1 / 2} \odot$ as a function of the rotation period. As mentioned above, the detection completeness for the rotation period of the stars with the starspot area $<10^{-3} A_{1 / 2} \odot$ is almost zero due to the detection limit. Since it is difficult to estimate the correct total number of the stars in a given period bin, here we used the number of the stars whose starspot area $>10^{-3} A_{1 / 2} \odot$ 


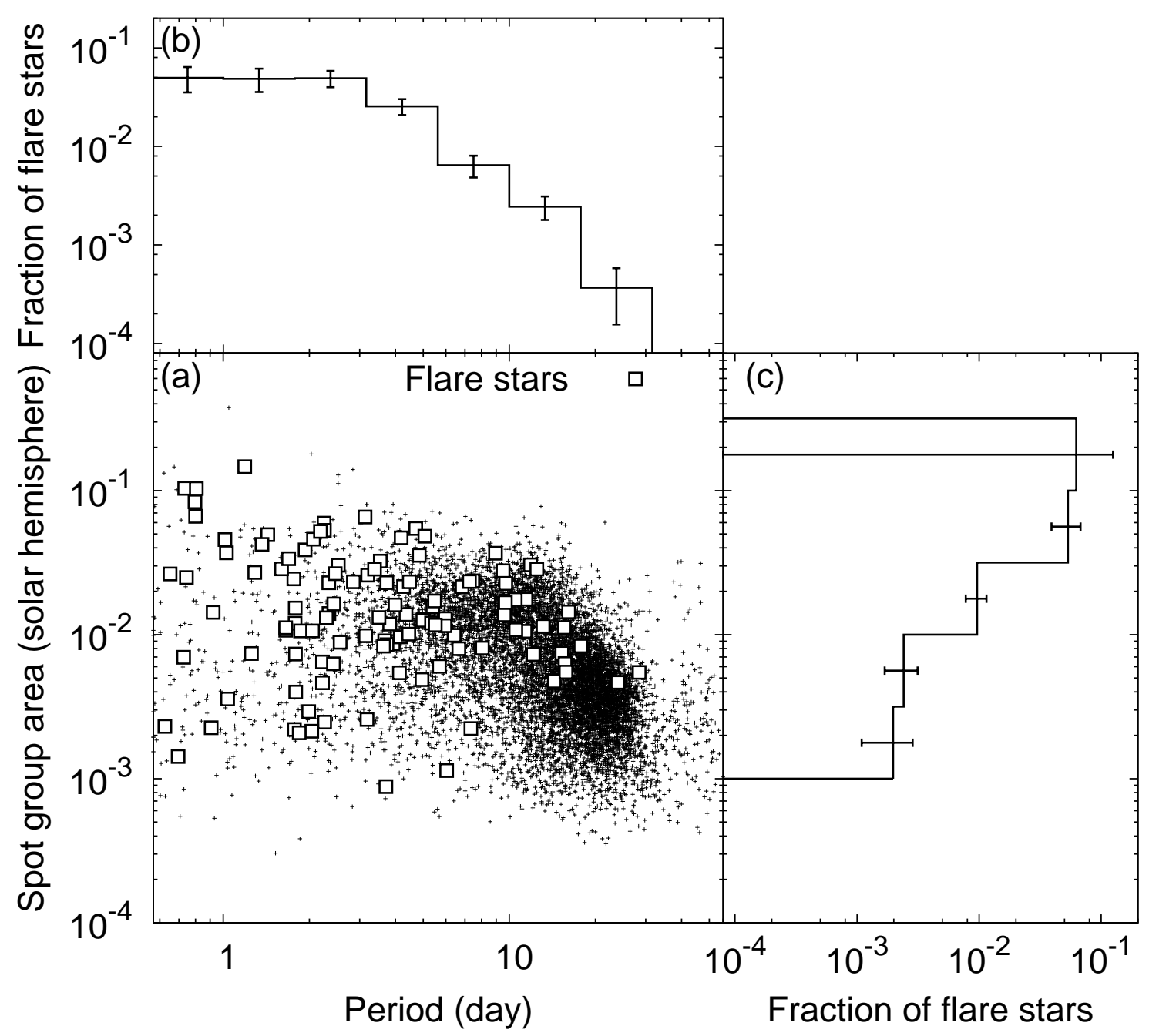

Fig. 1. (a) Scatter plot of the spot group area of solar-type stars as a function of the rotation period. The vertical axis represents the total area of starspots (in units of the area of solar hemisphere $\left(A_{1 / 2} \odot \sim 3.3 \times 10^{22} \mathrm{~cm}^{2}\right)$ estimated from the amplitude of quasi-periodic light variations by using equation (3). Open squares and small dots indicate solar-type stars with and without superflares respectively. (b) Histogram of the fraction of the stars showing superflares as a function of rotation period. The error bars represent the square root of the number of flare stars in each bin. (c) Same as (b) but as a function of the spot group area. 


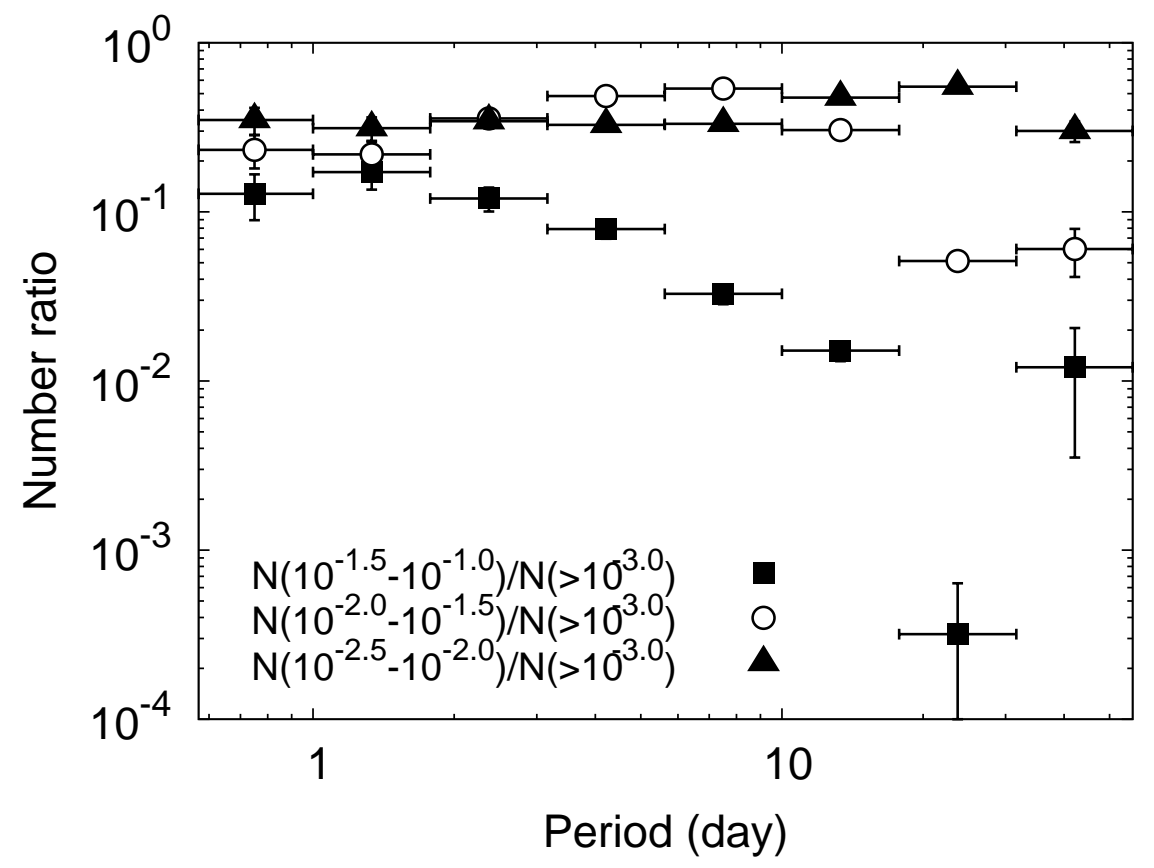

Fig. 2. The fraction of the stars with large starspots among the stars whose area of spots is larger than $10^{-3} A_{1 / 2 \odot}$. Filled squares, open circles, and filled triangles indicate the stars whose starspot area are $10^{-1.5}-10^{-1.0}, 10^{-2.0}-10^{-1.5}$, and $10^{-2.5}-10^{-2.0} A_{1 / 2 \odot}$, respectively. The horizontal and vertical error bars represent the bin width and the square root of the number of stars in each period bin respectively.

instead of the total number of stars in a given period bin. The fraction of the stars with the starspot area of $10^{-1.5}-10^{-1.0} A_{1 / 2} \odot$ (filled squares) decreases as the rotation period increases in the period range above 3 days. On the other hand, the fractions of the stars with the starspot area of $10^{-2.0}$ $10^{-1.5} A_{1 / 2} \odot$ (open circles) and $10^{-2.5}-10^{-2.0} A_{1 / 2} \odot$ (filled triangle) are almost constant in the ranges of the rotation period of $0.6<P_{\text {rot }}<20$ days and $0.6<P_{\text {rot }}<60$ days, respectively .

\subsection{Fraction of superflare stars as a function of rotation period}

Figure 3 shows the fraction of the stars showing superflares with the bolometric energy of $>10^{34} \mathrm{erg}$ among the stars with a given starspot area as a function of rotation period. The fractions of superflare stars among the stars with the starspot area of $10^{-1.5}-10^{-1.0} A_{1 / 2 \odot}$ (filled squares), $10^{-2.0}-10^{-1.5}$ $A_{1 / 2 \odot}$ (open circles) and $10^{-2.5}-10^{-2.0} A_{1 / 2} \odot$ (filled triangles), are roughly constant in the period range of $<3$ days. However, the observed fraction of superflare stars decreases as the rotation period increases in the period range of $>3$ days. This indicates that the large amount of the slowly-rotating stars with the moderately large starspots $\left(A_{\text {spot }} \sim 10^{-2} A_{1 / 2} \odot\right)$ did not exhibit energetic superflares during the Kepler observations. 


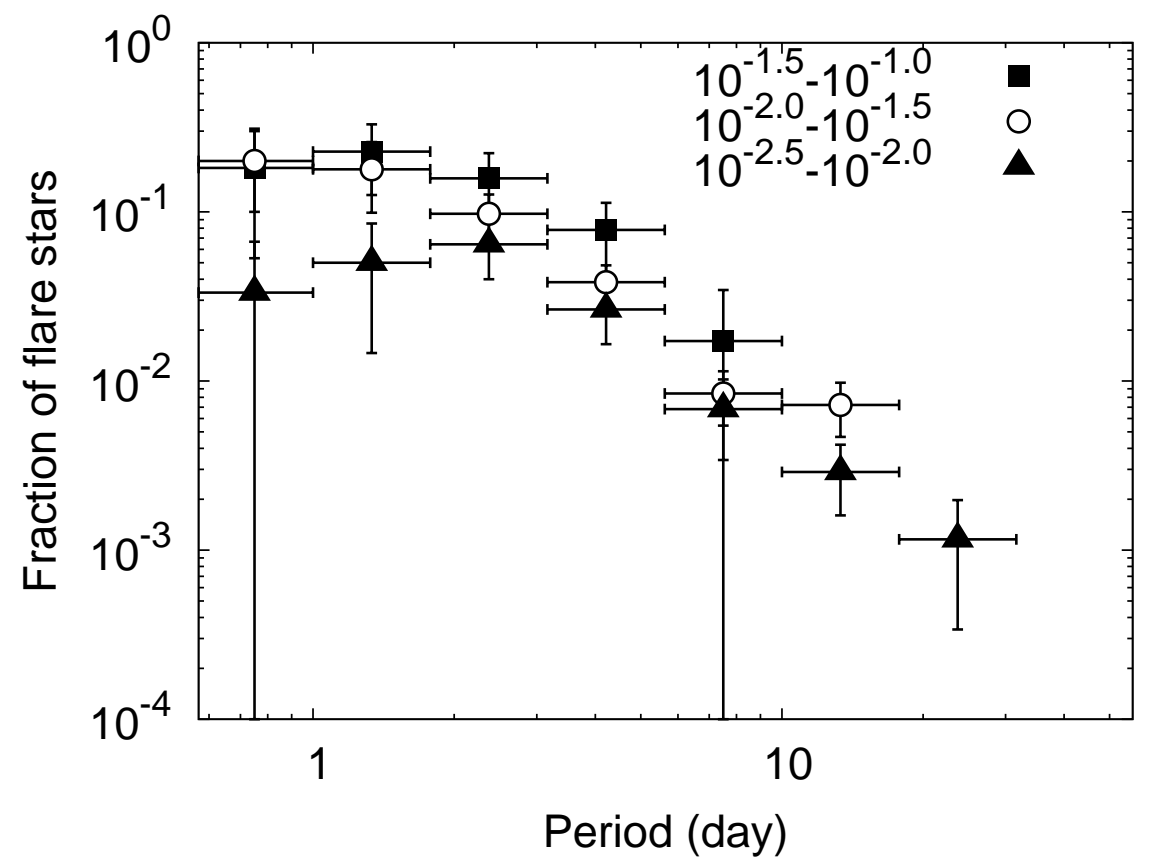

Fig. 3. The fraction of superflare stars (stars showing superflares with $E_{\mathrm{flare}}>10^{34} \mathrm{erg}$ during the Kepler observations) among the stars with a given spot area. Filled squares, open circles and filled triangles indicate the flare star fraction among the stars with the starspot area of $10^{-1.5}-10^{-1.0}, 10^{-2.0}-10^{-1.5}$ and $10^{-2.5}-10^{-2.0} A_{1 / 2 \odot}$, respectively. The horizontal and vertical error bars represent the bin width and the square root of the number of superflare stars in each period bin respectively.

\section{Discussion}

\subsection{Frequency of large starspots on slowly-rotating stars}

Figure 4 shows the cumulative fraction of the stars with a given spot area in each period range. The cumulative fraction was calculated as the ratio of the number of stars whose starspots area are larger than or equal to a given value to the total number of stars in each period range. The cumulative number of stars becomes almost constant in the spot area range below $10^{-3} A_{1 / 2 \odot}$. As mentioned in the previous section, this caused by that the periodic light variations the stars with small starspots could not be detected. The fraction of the stars rapidly decreases as the area of starspots increases in the range of starspot area above $10^{-2.5}-10^{-2.0} A_{1 / 2 \odot}$. The fraction of the stars with a given starspot area increases as the rotation period decreases. These results indicate that the average magnetic activity on rapidly-rotating stars is higher than that on slowly-rotating stars.

If we assume that (1) the typical lifetime of starspot groups is longer than the rotation period of the stars, and (2) most of our samples have the similar stellar properties, the number of stars with a give starspot area would be proportional to the appearance frequency of starspots with a given area. Under these assumptions, the number distribution of the stars as a function of the starspot area would correspond to the appearance frequency distribution of the starspots as a function of the starspot area. 


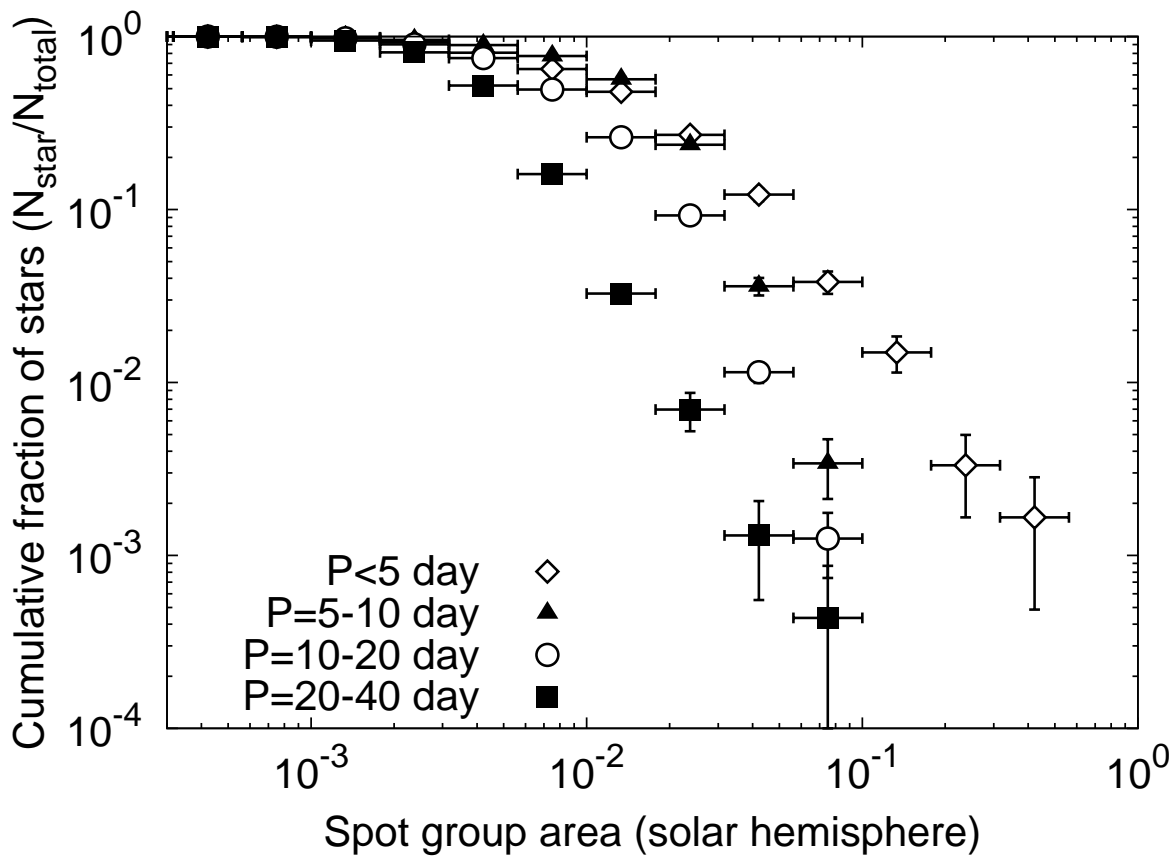

Fig. 4. Cumulative fraction of stars as a function of the area of starspots. The vertical axis indicates the number of stars with the given spot area and rotation period range normalized by the total number of stars in the given period range. Open diamonds, filled triangles, open circles and filled squares represent the cumulative fraction of solar-type stars with the rotation period of $<5$ days, 5-10 days, 10-20 days, and 20-40 days. The vertical and horizontal error bars indicate the square root of the number of stars and bin width respectively.

According to Petrovay \& van Driel-Gesztelyi (1997), the lifetime of sunspots increases as the area of sunspot increases. If the relation between lifetime of the spot and spot area in the Sun is similar to that in the solar-type stars, the lifetime of large starspots $\left(A>10^{-3} A_{1 / 2} \odot\right)$ is longer than the mean rotation period of the stars in this analysis $\left(\overline{P_{\text {rot }}} \sim 30\right.$ days $)$. As shown in appendix 1 , the solartype stars with large amplitude $(\sim 1 \%)$ photometric variations have large starspots with the area of the order of $\sim 10^{-2} A_{1 / 2} \odot$ and the lifetime of such large spots ranges from $\sim 50-\sim 300$ days which is longer enough than the rotation period of the star. We compared the frequency-area distribution of starspots on slowly-rotating solar-type stars $\left(P_{\text {rot }}=20-40\right.$ days $)$ and that of sunspot groups. As described in section 2.4, since the area of starspots were derived from the median of the amplitude of light variations within one rotation period taken from McQuillan et al. (2014), we can estimate only the typical size of the largest starspot group on each star. Let $N_{\text {star }}(A)$ be a number of stars with the starspot area of $\geq A$. The fraction of the stars with the starspot area in excess of $A, N_{\text {star }}(A) / N_{\text {star,total }}$, is equivalent to the expected number of the largest starspots with the area of $\geq A$ per star during one rotation period.

As mentioned in the section 3.1, it is difficult to estimate the correct number of $N_{\text {star,total }}$ from the data set because the period and amplitude of rotational light variations of the stars with small 
starspots could not be detected. We estimated the total number of stars with the rotation period of 20-40 days from the empirical gyrochronology relation (e.g., Barnes 2007; Mamajek \& Hillenbrand 2008; Meibom et al. 2009; Meibom et al. 2015). Let $N_{\text {star,total }}(P)$ to be the number of stars with the rotation period of $\geq P$ in the sample. $N_{\text {star,total }}(P)$ can be estimated from the duration of the main sequence phase $\left(\tau_{\mathrm{MS}}\right)$, the gyrochronological age of the star $\left(t_{\text {gyro }}(P)\right)$, and the total number of the samples $\left(N_{\text {all }}\right)$ as follows:

$$
N_{\text {star,total }}(P)=\left(1-\frac{t_{\text {gyro }}(P)}{\tau_{\mathrm{MS}}}\right) N_{\text {all }},
$$

if we assume that the star formation rate around the Kepler field has been roughly constant for $\tau_{\mathrm{MS}}$. According to Mamajek \& Hillenbrand (2008), the age of the star with the temperature of $\sim 5800 \mathrm{~K}$ ( $B-V \sim 0.65$ ) and rotation period of $>20$ days is $>2.9 \pm 0.5$ Gyr. This suggests that $\sim 70 \pm 10 \%$ of the stars with the temperature of $\sim 5800 \mathrm{~K}$ have the rotation period longer than 20 days, and $N_{\text {star,total }}$ for solar-type stars with $P_{\text {rot }}=20-40$ days in our sample would be $\sim 45,000 \pm 5000$. As mentioned above, the typical lifetime of the spots with the area of $>10^{-3} A_{1 / 2} \odot$ is thought to be longer than the mean rotation period of the star $\left(\overline{P_{\text {rot }}} \sim 30\right.$ days $)$. This suggests that we can ignore the appearance and disappearance of starspots during one rotation period which would affect the the appearance frequency of starspots. Therefore the appearance frequency of starspot groups (the average number of starspot groups per star per unit time) with the area of $\geq A$ averaged over one rotation period can be written as

$$
\bar{N}_{\text {starspot }}(A)=\frac{N_{\text {star }}(A)}{\overline{P_{\text {rot }}} N_{\text {star,total }}} .
$$

The appearance frequency of sunspot groups with the maximum area of the sunspot group of $\geq A$ can be written as

$$
\bar{N}_{\text {sunspot }}(A)=\frac{N_{\text {sunspot }}(A)}{T_{\text {obs,total }}},
$$

where $N_{\text {sunspot }}(A)$ and $T_{\text {obs,total }}$ are the number of sunspot groups with the maximum area of $\geq A$ and the total time span of observations respectively. We analyzed the Royal Observatory, Greenwich - USAF/NOAA Sunspot Data between 1874 and 2015 ${ }^{1}$ and calculated the appearance frequency of sunspot groups as a function of the sunspot group area.

Figure 5 represents the comparison between the cumulative appearance frequency $(\bar{N}(A))$ of starspots on solar-type stars with the rotation period of 20-40 days (solid lines) and that of sunspot groups (dotted lines). The occurrence frequency of decreases as the area of sunspots increases. The both appearance rate of the sunspot groups and that of starspots with the area $>10^{-2.5} A_{1 / 2} \odot$ is approximately once in a few years. The cumulative appearance frequency $(\bar{N}(A))$ of starspots on solar-type

\footnotetext{
${ }^{1}$ The data are retrieved from http://solarscience.msfc.nasa.gov/greenwch.shtml.
} 


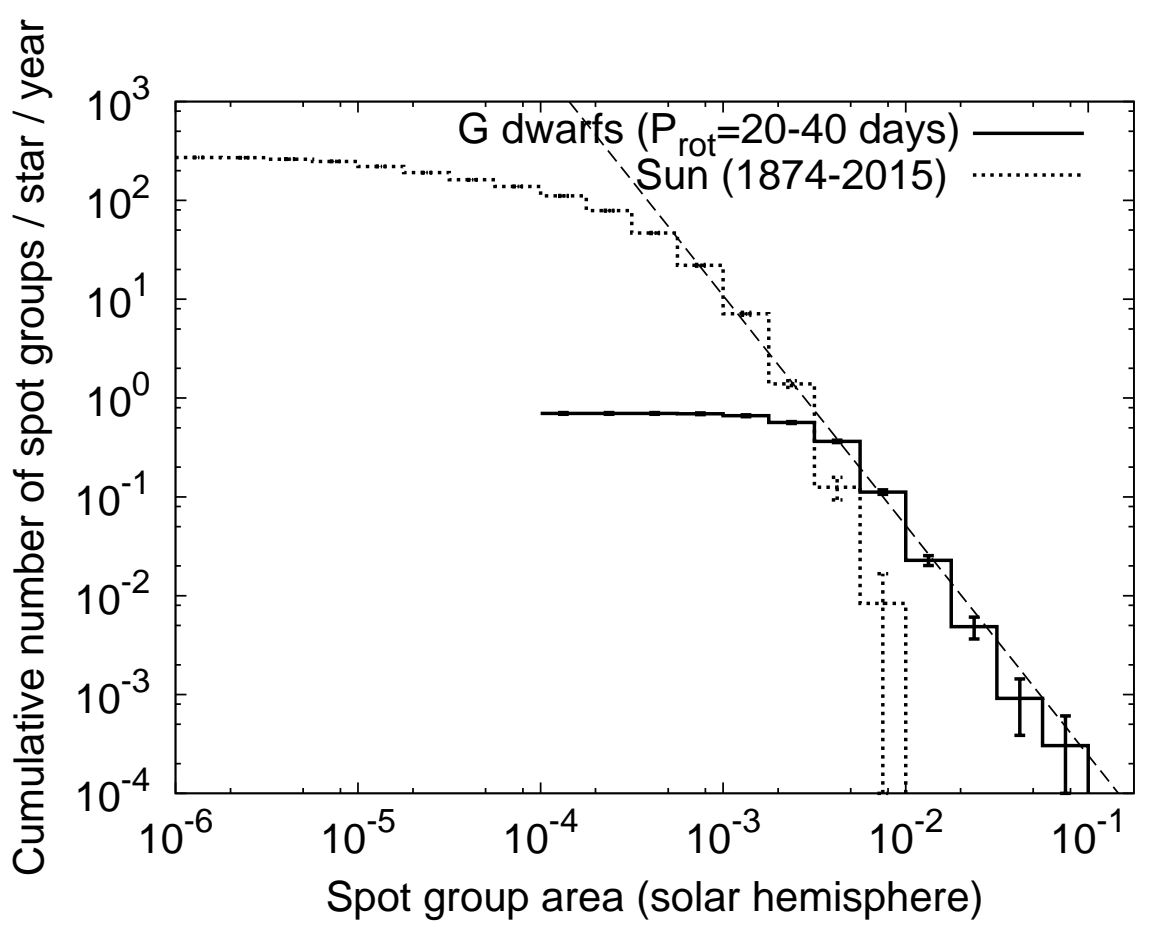

Fig. 5. Comparison between the appearance frequency - spot area distribution of starspots on slowly-rotating solar-type stars and that of sunspot groups. Solid lines indicate the cumulative appearance frequency of starspots on the solar-type stars with the rotation period of 20-40 days. Dotted lines indicate the cumulative appearance frequency of sunspot groups as a function of the maximum area of each sunspot group. The thin dashed line represents the power-law fit to the frequency distribution of starspots in the spot area range of $10^{-2.5}-10^{-1.0} A_{1 / 2 \odot}$. The power-law index of the line is $-2.3 \pm 0.1$.

stars can be fitted by a power-law function with the power-law index of $-2.3 \pm 0.1$ (dashed line) for the spot area between $10^{-2.5}$ to $10^{-1.0}$. According to Bogdan et al. (1988), the size distribution of individual sunspot umbral areas shows the lognormal distribution. Although, the overall cumulative appearance frequency-size distribution of sunspot groups also shows the similar lognormal distribution, the size distribution of sunspot groups for large sunspots is roughly on this power-law line for the spot area between $10^{-3.5} A_{1 / 2 \odot}$ to $10^{-2.5} A_{1 / 2 \odot}$. The appearance frequency of sunspots with the spot area of $\sim 10^{-2} A_{1 / 2 \odot}$ is about 10 times lower than that of starspots on solar-type stars. This difference between the Sun and solar-type stars might be caused by the lack of "super-active" phase on our Sun during the last 140 years (e.g., Schrijver et al. 2012). The similarity between the size distribution of sunspots and that of starspots implies that the both sunspots and larger starspots might be produced by the same physical process.

\subsection{Frequency of superflares and starspot area}

As shown in figure 3, the fraction of the stars showing superflares increases as the area of starspots increases. This suggests that the average occurrence frequency of superflares increases as the starspot area increases. In addition to the fraction of flare stars and flare frequency, the energy released by the 


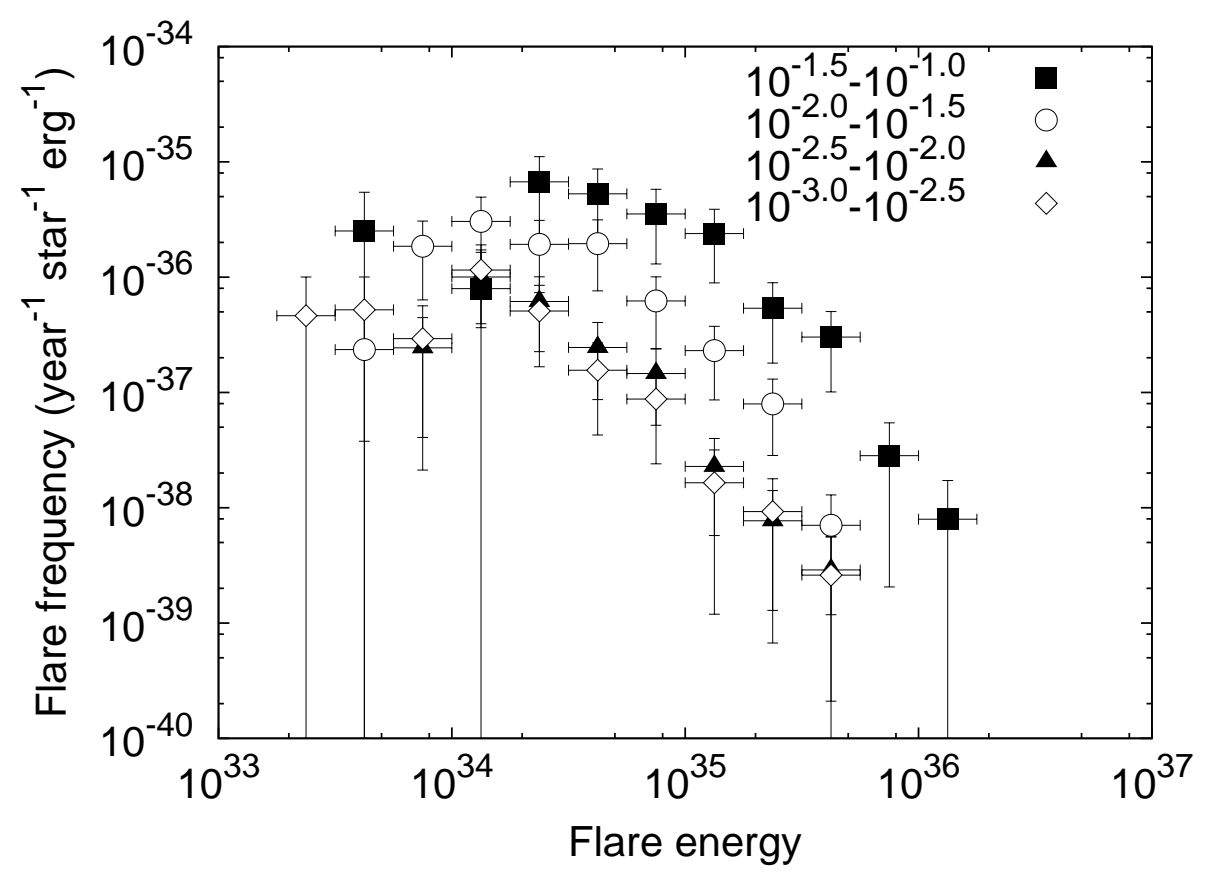

Fig. 6. Occurrence frequency distribution of superflares on solar-type stars with different starspot area. Filled squares, open circles, filled triangles and open diamonds represent the flare frequency on solar-type stars with starspot area of $10^{-1.5}-10^{-1.0}, 10^{-2.0}-10^{-1.5}, 10^{-2.5}-10^{-2.0}$, and $10^{-3.0}$. $10^{-2.5} A_{1 / 2} \odot$, respectively. The vertical error bars indicate the square-root of the number of detected flares. The horizontal error bars indicate the bin width.

largest superflare also increases as the area of starspots increases (e.g., Shibata et al. 2013; Maehara et al. 2015). Figure 6 represents the frequency distribution of superflares on solar-type stars with different starspot area as a function of the energy released by flares. The frequency of superflares on solar-type stars with a given starspot area is calculated from the number of solar-type stars with a given starspot area, the total time span of observations, and the number of flares which occurred on the solar-type stars with a given starspot area.

The frequency of superflares with a given flare energy clearly increases as the area of starspots increases. The larger end of the frequency distribution is also increases as the starspot area increases. The larger end of the frequency distribution of superflares on solar-type stars with the spot area of $\sim 10^{-2.5} A_{1 / 2} \odot$ is $\sim 3 \times 10^{35} \mathrm{erg}$ and that on the stars with the spot area of $\sim 10^{-1.5} A_{1 / 2} \odot$ is $\sim 10^{36}$ erg.

We adopted the same analysis to solar flares. We used the event list of solar flares observed with the GOES satellite from 1976 to $2015^{2}$. Combined with the list of solar flares and the Royal Observatory, Greenwich - USAF/NOAA Sunspot Data, we calculated the frequency of solar flares on active regions (ARs) with a given sunspot area from the number of flares on ARs, the number $\times$

\footnotetext{
${ }^{2}$ The data are retrieved from ftp://ttp.ngdc.noaa.gov/STP/space-weather/solar-data/solar-features/solar-flares/x-rays/goes/xrs/.
} 


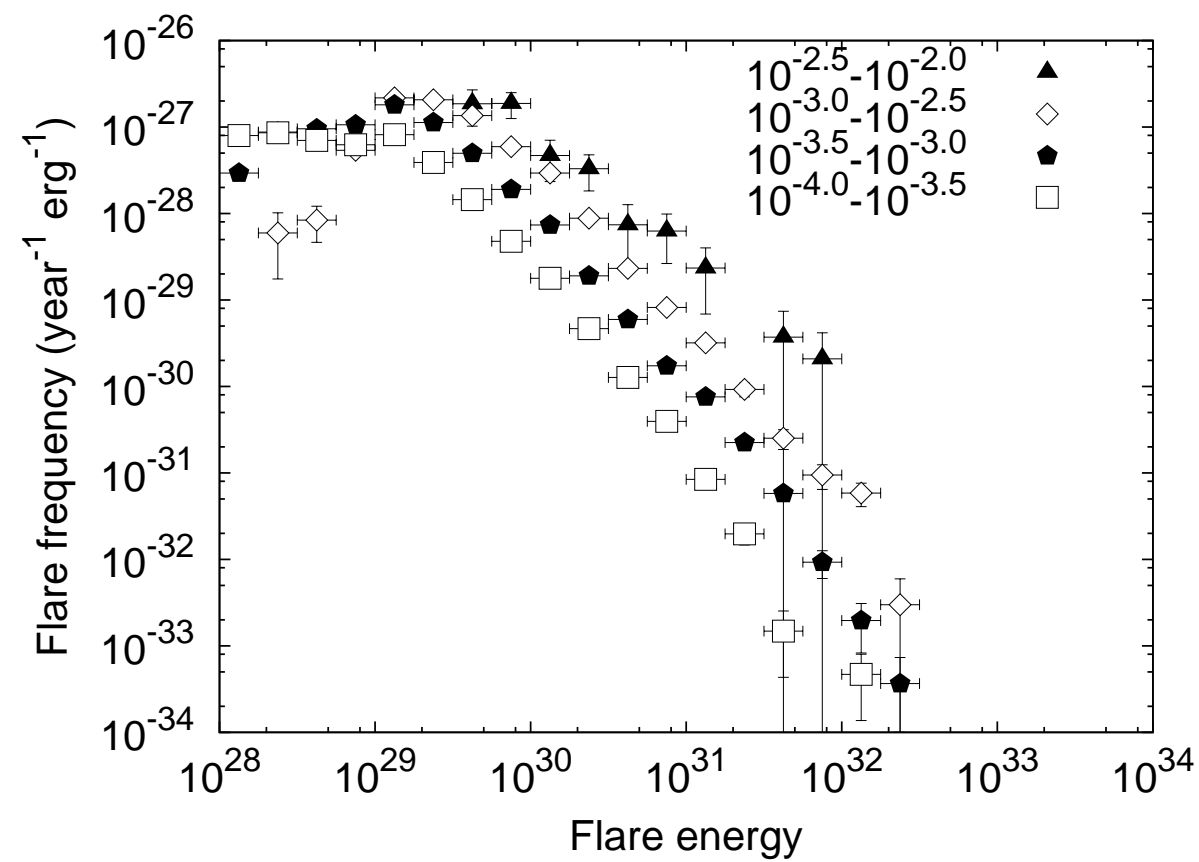

Fig. 7. Occurrence frequency distribution of solar flares on active regions (ARs) with different sunspot group area. Filled triangles, open diamonds, filled pentagons, and open squares represent the flare frequency on ARs with sunspot group area of $10^{-2.5}-10^{-2.0}, 10^{-3.0}-10^{-2.5}, 10^{-3.5}-10^{-3.0}$, and $10^{-4.0}-10^{-3.5} A_{1 / 2} \odot$, respectively.

duration of ARs with a given area. We assumed that bolometric energies of B, C, M, X, and X10 class solar flares are $10^{28}, 10^{29}, 10^{30}, 10^{31}$, and $10^{32}$ erg from observational estimates of energies of typical solar flares (e.g., Benz 2008; Emslie et al. 2012). Figure 7 shows the frequency distribution of solar flares on ARs with different sunspot group area. The occurrence frequency of flares increases as the sunspot group area increases.

Figure 8 shows the comparison between the occurrence frequency distribution of superflares on solar-type stars (open circles and open diamonds) and that of solar flares (filled diamonds and filled squares). The solid line indicates the power-law fit to the frequency distribution of solar flares on ARs with sunspot group area of $10^{-3.0}-10^{-2.5} A_{1 / 2} \odot$ between $10^{30} \mathrm{erg}$ and $10^{33} \mathrm{erg}$. The power-law index is $-1.99 \pm 0.05$. Both the occurrence frequency distribution of superflares on solar-type stars with the starspot area of $10^{-3.0}-10^{-2.5} A_{1 / 2 \odot}$ (open diamonds) and that of solar flares on ARs with the same sunspot group area $\left(10^{-3.0}-10^{-2.5} A_{1 / 2 \odot}\right)$ are roughly on the same power-law line. Dashed and dotted lines in figure 8 indicate power-law distribution with the same power-law index but 10 and 1/10 times frequency of the solid line. The frequency distribution of solar flares on ARs with sunspot group area of $10^{-4.0}-10^{-3.5} A_{1 / 2 \odot}$ and that of superflares on solar-type stars with the starspot area of $10^{-2.0}-10^{-1.5} A_{1 / 2 \odot}$ are roughly on the dotted and dashed lines respectively. These results suggest that both the frequency of flares is roughly proportional to the area of spot groups. 


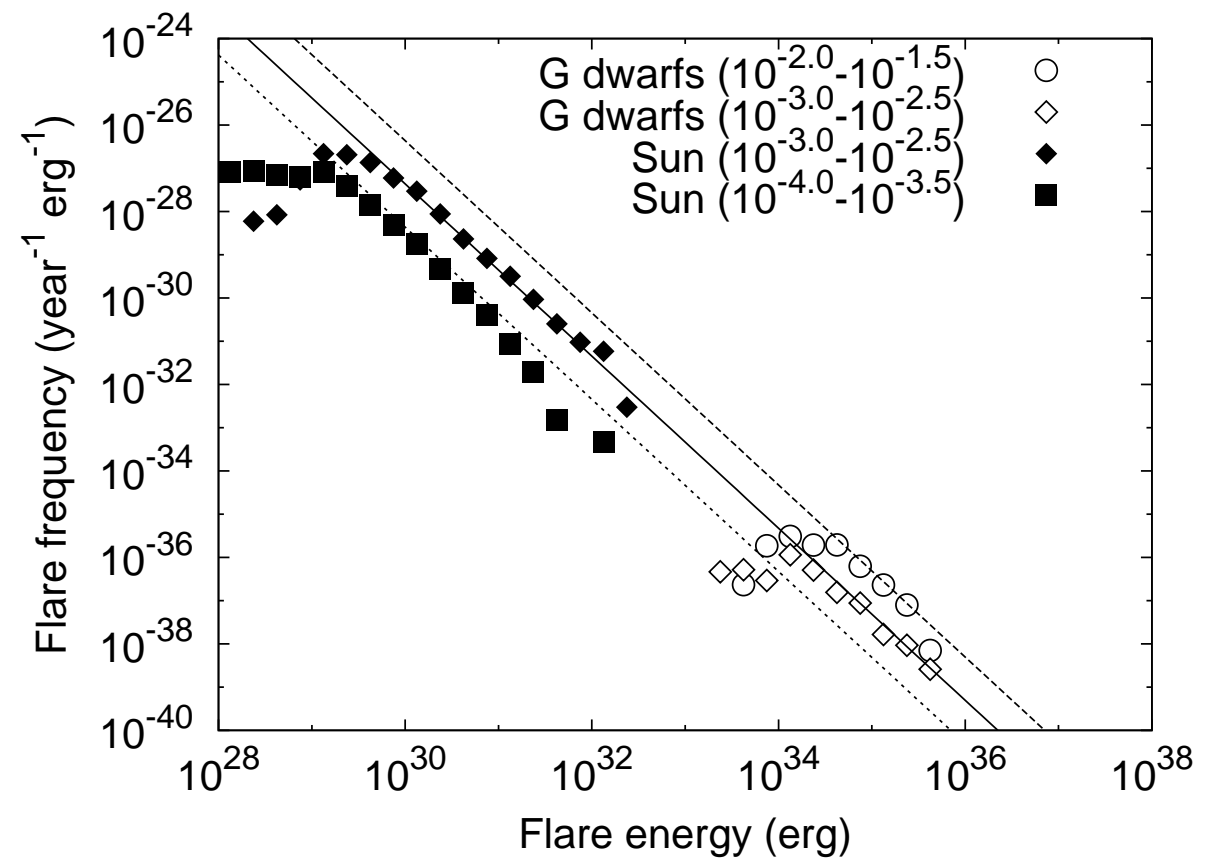

Fig. 8. Comparison between occurrence frequency distribution of superflares on solar-type stars and that of solar flares. Open circles and open diamonds represent the frequency of superflares on solar-type stars with spot area of $10^{-2.0}-10^{-1.5}$ and $10^{-3.0}-10^{-2.5} A_{1 / 2} \odot$. Filled diamonds and filled squares represent the frequency of solar flares on active regions (ARs) with the sunspot group area of $10^{-3.0}-10^{-2.5}$ and $10^{-4.0}-10^{-3.5} A_{1 / 2} \odot \cdot$ Solid line indicates the power-law fit to the frequency of solar flares on ARs with sunspot group area of $10^{-3.0}-10^{-2.5} A_{1 / 2} \odot$ between $10^{30}$ erg and $10^{33}$ erg. The power-law index is $-1.99 \pm 0.05$. Dashed and dotted lines are the power-law distributions with the same power-law index but 10 - and $1 / 10$ - times flare frequency of the solid line.

\subsection{Flare activity and magnetic structure of spot groups}

The flare activity correlates with not only the area of sunspot groups, but also magnetic structure of sunspot groups (e.g., Bell \& Glazer 1959; Sammis et al. 2000). We performed the same analysis as in figure 7 for solar flares on different magnetic classifications. The magnetic classifications of each sunspot group were taken from the USAF/NOAA Sunspot Data observed between 1981 and $2015^{3}$. Figure 9 represents the frequency distribution of solar flares on ARs with the same sunspot area but different magnetic structures. As previous studies show, the occurrence frequency of flares increases as the magnetic structure of the sunspot groups becomes complicated. The frequency of flares with a given flare energy on the bipolar ( $\beta$-, $\gamma-$, and $\delta$-type) sunspot groups is 1-2 orders of magnitude higher than that on the unipolar ( $\alpha$-type) sunspot groups. $\delta$-type sunspot groups exhibit more frequent flares than $\beta$ - and $\gamma$-type sunspot groups. The magnetic structure of sunspot groups also has a relation to the productivity of large solar flares. According to Sammis et al. (2000), more than $80 \%$ of X-class flares occurred on $\delta$-type spot groups. As shown in figure 9, the largest end of the frequency-energy

\footnotetext{
${ }^{3}$ The data are retrieved from ftp://ftp.ngdc.noaa.gov/STP/SOLAR_DATA/SUNSPOT_REGIONS/USAF_MWL/.
} 
distribution on $\delta$-type sunspot groups is also larger than those on other types of sunspot groups with the same spot area.

As mentioned in section 3.2, the observed fractions of the stars showing superflares with the energy $>10^{34} \mathrm{erg}$ among the stars with the starspot area of $10^{-1.5}-10^{-1.0}, 10^{-2.0}-10^{-1.5}$, and $10^{-2.5}$ $10^{-2.0} A_{1 / 2} \odot$ decreases as the rotation period increases. Since the total time span of the observations for the detection of superflares is limited ( $\sim 480$ days), the observed fraction of superflare stars can be affected by the frequency of superflares. Figure 10 represents the frequency of superflares on each superflare star as a function of the rotation period. The average flare frequency on the rapidly-rotating superflare stars ( $P_{\text {rot }}=2-3$ days) is a few times higher than that on the superflare stars with $P_{\text {rot }}>6$ days. The rate of decrease in the frequency of superflares as a function of rotation period is smaller than that in the fraction of stars vs. rotation period. It should be noted that Davenport (2016) reported many flare stars with the rotation period below 2-3 days and some of them are not listed in Shibayama et al. (2013). On the other hand, only a few flare stars with $P_{\text {rot }}>3$ days are listed as a flare star by Davenport (2016) as mentioned in appendix 2. This difference may be caused by the differences in the detection method and selection criteria. Since the flare detection threshold used in Shibayama et al. (2013) depends on the rotation period and some superflares on rapidly-rotating stars may be missed, the flare frequency on rapidly-rotating superflare stars may be underestimated.

The frequency-energy distributions of superflares on the stars with different spots sizes (figure 6 and 8 ) indicate that the average frequency of superflares with the energy of $E_{\text {flare }}>10^{34}$ erg on the stars with the starspot area of $10^{-2.0}-10^{-1.5} A_{1 / 2 \odot}$ is $\sim 10^{-1}$ year $^{-1}$. The observed frequency of each flare star with $P_{\text {rot }}>6$ days is $\sim 1$ year $^{-1}$ (figure 10). Therefore, the fraction of flare stars expected from $\sim 500$ days observations would be $\sim 10^{-1}$. However, as shown in figure 3 , the observed fraction of the stars with superflares among the stars with the starspot area of $10^{-2.0}-10^{-1.5} A_{1 / 2} \odot$ and rotation period of $>6$ days is $\sim 10^{-2}$. This suggests that some of the stars with larger starspots and longer rotation period may show a much lower flare activity than the superflare stars with the same spot size. Moreover, the frequency of superflares on each star with the same spot size and rotation period have a large scatter ( $>1$ order of magnitude). These results imply that not only the existence of large starspots as previous studies pointed out but also other factors may play important roles in generation of superflares. By analogy with the correlation between the flare activity and the magnetic structure of sunspot groups, one of the possible explanation for the difference in flare activity on the stars with the same spot area may be the difference in the magnetic structure of starspots. As shown in figure 9, both the flare frequency and the flare energy at the larger end of frequency-energy distribution of solar flares originated from the $\alpha$-type spots are 1-2 orders of magnitude lower than those from the $\delta$-type spots with the same spot area. If the correlation between the magnetic structure of large starspots 

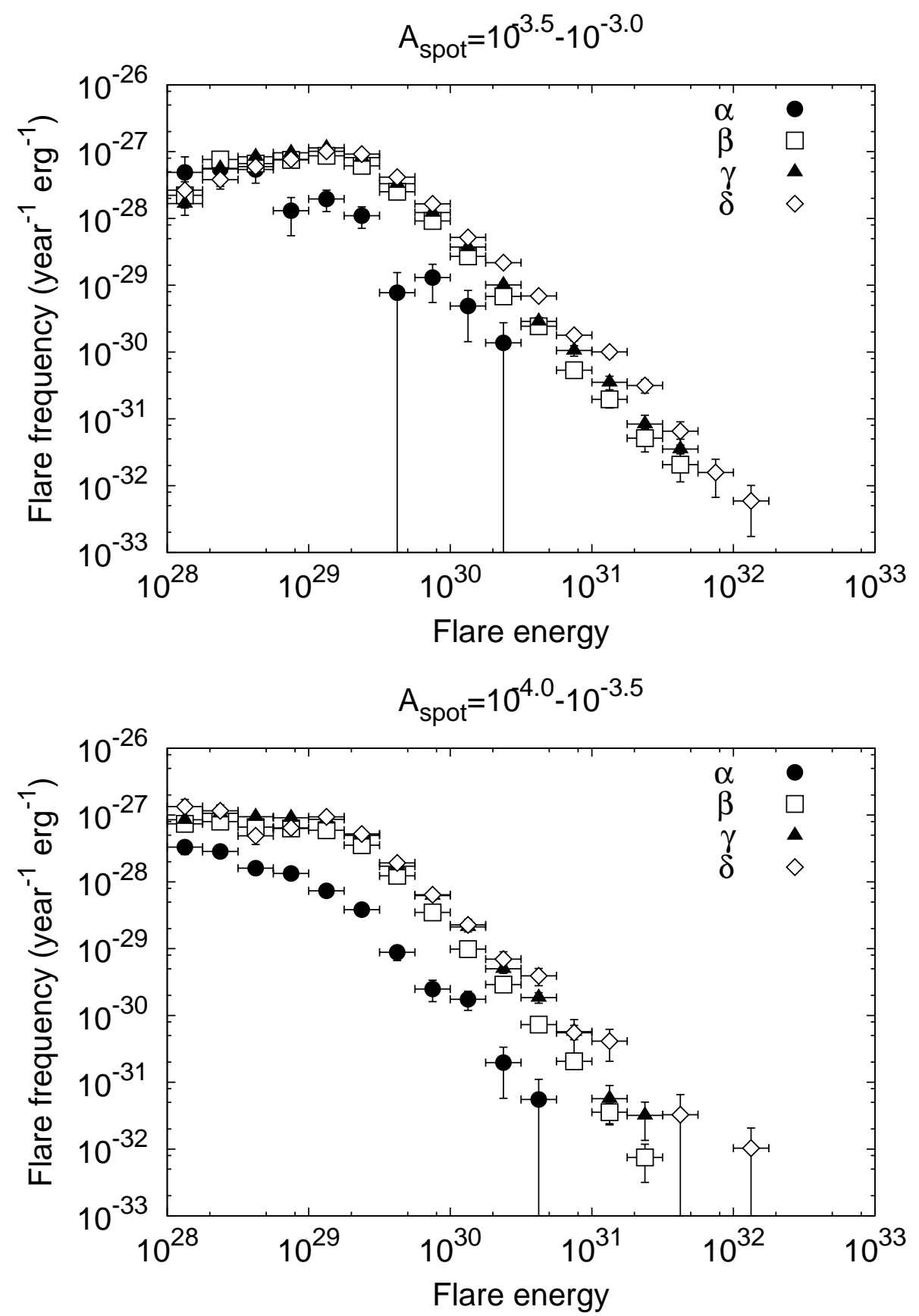

Fig. 9. Occurrence frequency distribution of solar flares on active regions (ARs) with different magnetic classifications. Upper and lower panels show frequency distribution of solar flares on active regions (ARs) with the sunspot group area of $10^{-4.0}-10^{-3.5}$ and $10^{-3.5}-10^{-3.0} A_{1 / 2 \odot}$. Filled circles, open squares, filled triangle, and open diamonds represent the flare frequency on $\alpha, \beta, \gamma(\gamma+\beta \gamma)$, and $\delta(\beta \delta+\gamma \delta+\beta \gamma \delta)$-type sunspot groups, respectively. The horizontal error bars indicate the bin width and the vertical error bars correspond to the square root of the number of flares. 


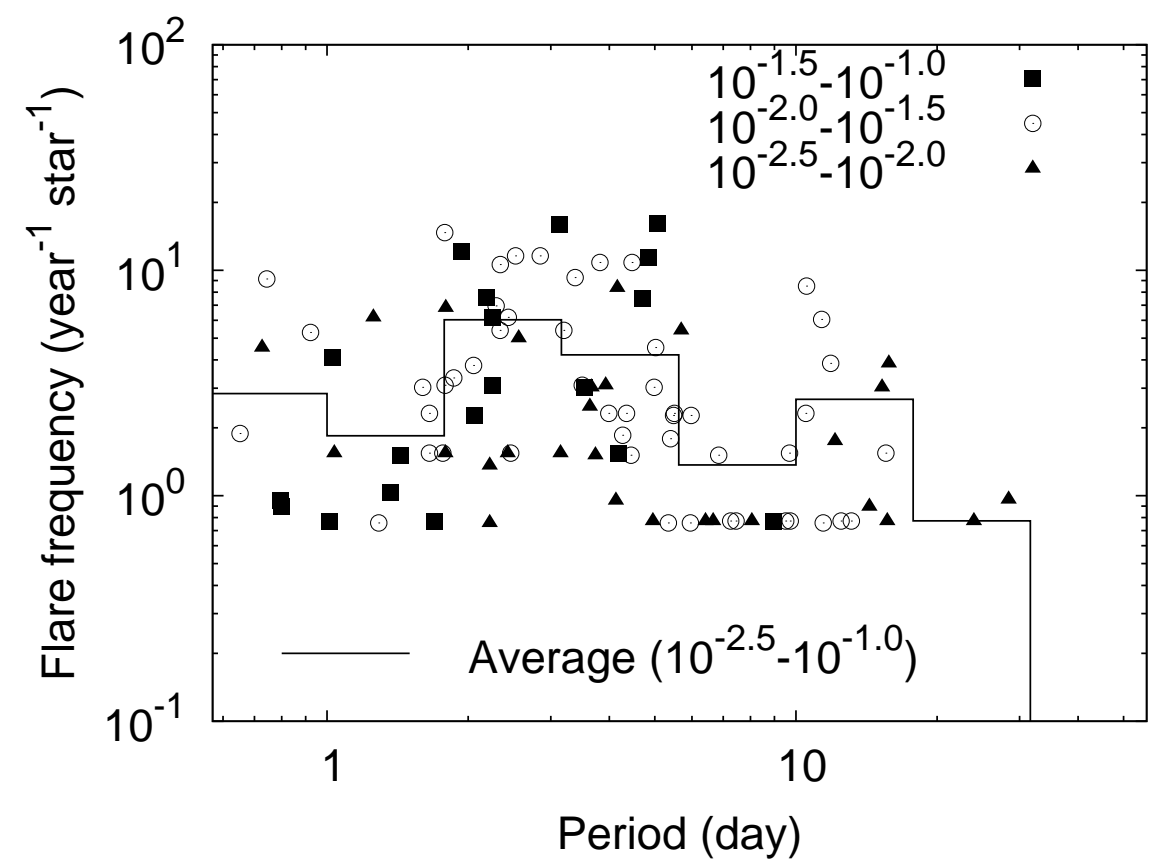

Fig. 10. The frequency of superflare with $E_{\mathrm{flare}}>10^{34} \mathrm{erg}$ on the superflare stars as a function of the rotation period. Filled squares, open circles, and filled triangles indicate the frequency of superflares $\left(E_{\mathrm{flare}}>10^{34} \mathrm{erg}\right)$ on each superflare star with the area of starspots of $10^{-1.5}-10^{-1.0}, 10^{-2.0}-10^{-1.5}$, and $10^{-2.5}-10^{-2.0} A_{1 / 2 \odot}$, respectively. The solid line histogram represents the average frequency of superflares on superflare stars with the area of starspots $>10^{-2.5} A_{1 / 2 \odot}$. Since the typical length of observation period for the flare detection is $\sim 480$ days, the lower limit of the flare frequency is $\sim 1$ event/480days $\sim 0.76$ year $^{-1}$.

and superflares on the solar-type stars is similar to that between the magnetic structure of smaller sunspots and solar flares on the Sun, the large starspots with the simple ( $\alpha$-type) magnetic structure would produce less frequent and less energetic flares than the large starspots with the complex $(\delta$ type) magnetic structure. According to sunspot observations (e.g., Sammis et al. 2000), majority of sunspot groups with large spot area are $\delta$-type sunspot group. The USAF/NOAA Sunspot Data indicate that the fraction of $\delta$-type spot groups among the sunspots with the area of $10^{-3.0}-10^{-2.5} A_{1 / 2} \odot$ is approximately $92 \%$. However, the small fraction of the stars with superflares among the stars with large starspots and longer rotation period (figure 3) suggests that if the difference in the flare activity of the stars with large starspots is caused by the difference in the magnetic structure of spots, the fraction of the $\delta$-type spots among the starspots with the area of $\sim 10^{-2.0} A_{1 / 2} \odot$ on the solar-type stars would be lower than that among the sunspots with the area of $\sim 10^{-3.0} A_{1 / 2 \odot}$. We need more detailed studies of the magnetic structure of large starspots on the stars with and without superflares. Doppler imaging (e.g., Rice 2002) and Zeeman Doppler imaging (e.g., Donati \& Landstreet 2009) techniques may help us to study the magnetic field geometry of the solar-type stars with large starspots and its correlation to the flare activity. The large starspots can cause the small fluctuations in the light curve 
of planetary transits (e.g., Silva 2003; Pont et al. 2007). The detailed analysis of the transit light curve with the future high photometric-precision and high time-resolution data (e.g., TESS; Ricker et al. 2015 and PLATO; Rauer et al. 2014) may also be useful to reveal the difference in the structure of the starspot groups between the stars with and without superflares.

\section{Acknowledgments}

Kepler was selected as the tenth Discovery mission. Funding for this mission is provided by the NASA Science Mission Directorate. The data presented in this paper were obtained from the Multimission Archive at STScI. This work was supported by MEXT/JSPS KAKENHI Grant Number 26800096, 26400231, 16H03955, 16J00320, and 16J06887. The authors wish to acknowledge the anonymous reviewer for his/her helpful comments to the manuscript.

\section{Appendix 1 Large starspots on solar-type stars}

As described in section 2, we estimated the area of starspot groups from the amplitude of the rotational light variations under the assumption that observed light variations are caused by one or a few large starspot group(s). In the case of rapidly-rotating stars, the existence of the large starspots has been studied by using the light curve modeling (e.g., Fröhlich et al. 2012; Notsu et al. 2013) and Doppler imaging technique (e.g., Strassmeier \& Rice 1998; Rice 2002). However, in the case of slowly-rotating stars, it is unclear whether the large amplitude light-variations are caused by a few large starspot groups or not. In order to check the assumption that light variations are caused by the large starspots, here we investigate the number of starspot groups on some of stars showing the light variations with large amplitude $(>1 \%)$ and long period ( $>20$ days).

In this analysis, we used a new simple method for identification of each starspot based on the rotational phase of local brightness minima in the light curve. The light curve of the rotating star with a starspot group shows a minimum brightness at the meridian passage of the spot during one rotation period. The time separation of each photometric minimum in the light curve is constant and equals to the rotation period. In the case of the star with two spot groups, the light curve shows two local minima during the rotation period. The separation of the two local minima corresponds to the difference in longitudes between the two spots. Moreover, if two spots are located at the different latitudes, the time separation of two local minima, which correspond to the the difference in longitudes between each spot group, changes in time due to the differential rotation of the star. Figure 11 shows some examples of the light curves of slowly-rotating stars (a, c, e, and g) and the time variations of the rotational phase of local brightness minima (b, $d, f$, and $h)$. All of the stars shown in figure 11 are non-superflare stars. The vertical axis of figure 11 (b, d, f, and h) represents the rotational phase of each local minimum in the light curve. The phase of each minimum were 
calculated by using the rotation period of the star taken from McQuillan et al. (2014). If a series of local minima are caused by the same spot group, the rotational phase of the local minima lie on a line on the phase-time plot until the spot group disappear. Therefore, we can identify each starspot and can estimate the lifetime of starspots by using our method. The slope of the line corresponds to the difference in the apparent rotation period of the spot group due to the differential rotation. This indicates that we can also measure the differential rotation in the stars from the standard deviation of the mean slope in the phase-time plots. In figure 11 (b), we can clearly see two series of the local minima between BJD 2455300 and 2455450. This result indicates that there have been two major starspot groups on the surface of KIC 8282807 during that period. If two or more spot groups are located at the same longitude, the amplitude of photometric variations would be large. As shown in figure 11, the two different spots were located at almost the same longitude around BJD 2455500 and then the amplitude of photometric variations became large. This effect causes the overestimation of the area of starspots by using the equation (3). However, if the latitudes of each spot are not the same, the relative longitudes between each spots change in time and we can estimate the area of each spot group from the light curve. The light curves and the phase-time plots of these slowly-rotating stars indicate that the number of major spot groups on the surface of the star at a given time is 1-3.

We also performed the same analysis for the light curves of the superflare stars with $P_{\text {rot }}=4$ 15 days. Figure 12 shows the light curves and phase-time plots of KIC 8482482 ( $P_{\text {rot }}=4.47$ days), KIC 10081606 ( $P_{\text {rot }}=7.46$ days $)$, KIC $10524994\left(P_{\text {rot }}=11.9\right.$ days $)$, and KIC $11972298\left(P_{\text {rot }}=15.6\right.$ days). The superflare stars with the short- $\left(P_{\text {rot }}<10\right.$ days $)$ and middle- $\left(P_{\text {rot }}=10-20\right.$ days $)$ rotation period also have a few major spots at the given time. These results indicate that the photometric amplitude can be used as a tracer of the spot area despite the rotation period and flare activity of the star.

The local photometric minima caused by the spot group lie on a line on the phase-time plot until the spot disappears. Thus we can estimate the lifetime of each spot group from the phasetime plots as shown in figure 11 and 12. These series of photometric local minima persist for a few hundred days (in the case of slowly-rotating stars) and $\sim 50-100$ days (rapidly-rotating stars), respectively. These results indicate that the typical lifetime of large starspot groups with the area of $\sim 10^{-2} A_{1 / 2} \odot$ on solar-type stars is longer enough than the rotation period of the star. In the case of slowly-rotating stars, the typical lifetime of large spots is the order of 100 days, which is much longer than the lifetime of the typical sunspot groups ( $<30$ days; e.g., Petrovay \& van Driel-Gesztelyi 1997). In section 4.2, we assumed that the lifetime of spot groups is longer than the rotation period. These results suggest that the assumption on the spot lifetime is basically valid for the large starspots. In case of the starspots with the area of $\sim 10^{-3} A_{1 / 2} \odot$, the lifetime would be comparable to large sunspot 

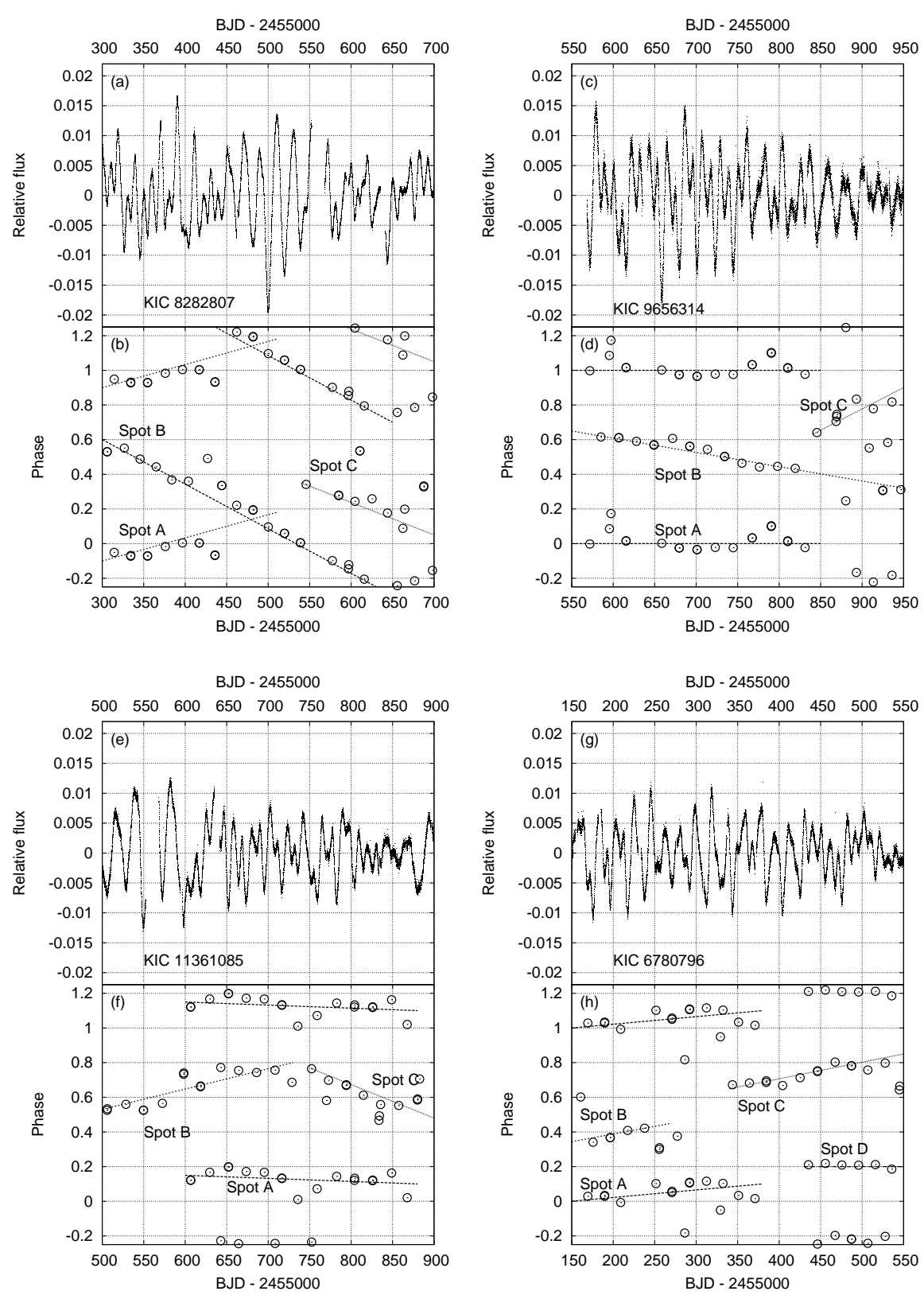

Fig. 11. (a): Light curve of the slowly-rotating solar-type star KIC 8282807 ( $P_{\text {rot }}=20.3$ days). (b): Time-variation of the rotational phase of the local photometric minima in the light curve of KIC 8282807. The rotational phase are calculated by using the rotation period taken from McQuillan et al. (2014). Open circles represent each local brightness minimum. Solid and dotted lines indicate each series of local minima caused by each spot group. (c) and (d): Same as panels (a) and (d), but for KIC 9656314 ( $P_{\text {rot }}=21.7$ days). (e) and (f): Same as panels (a) and (d), but for KIC 11361085 ( $P_{\text {rot }}=21.9$ days). (g) and (h): Same as panels (a) and (d), but for KIC 6780796 ( $P_{\text {rot }}=20.2$ days). 

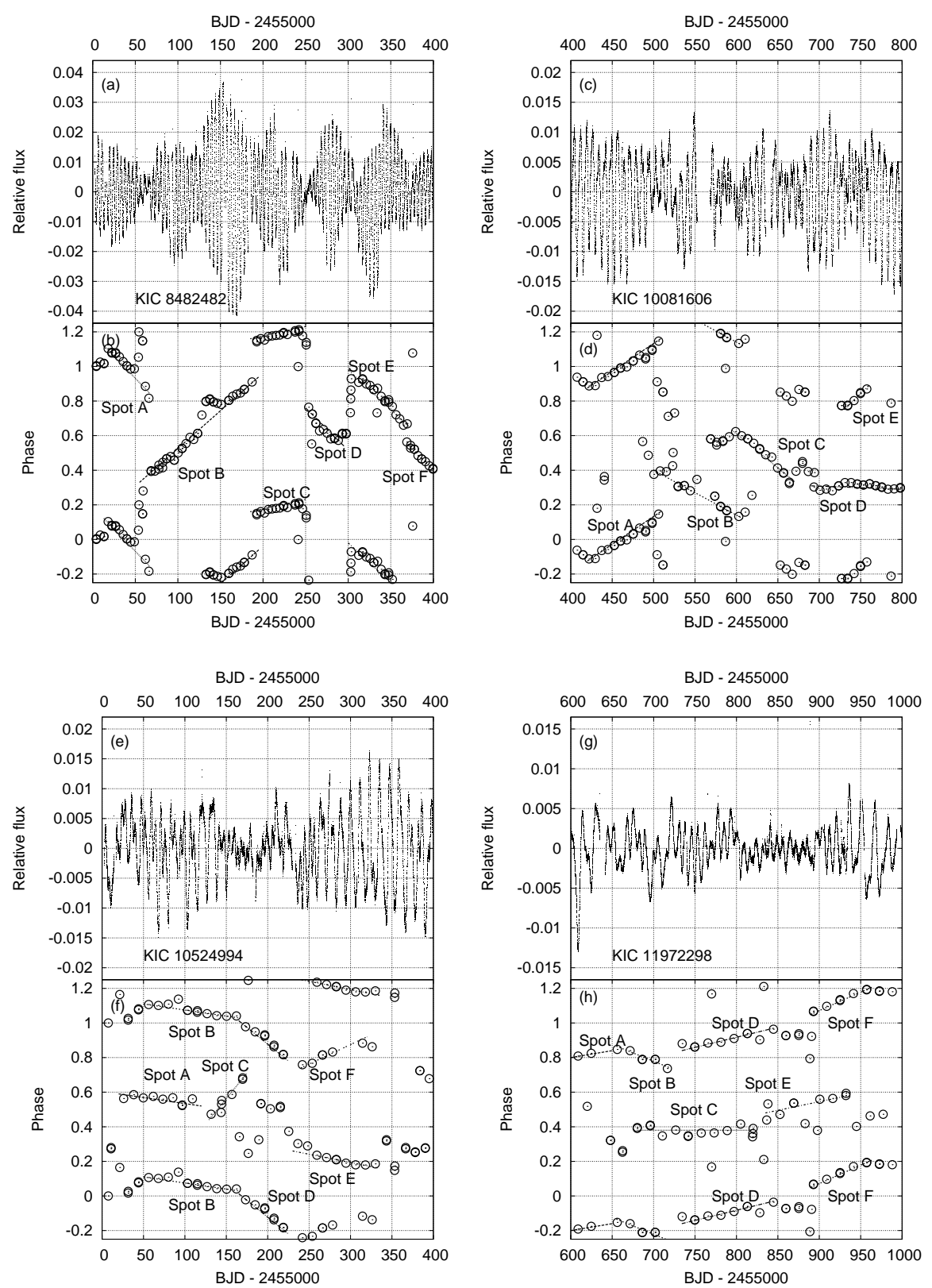

Fig. 12. (a): Light curve of the solar-type superflare star KIC 8482482 ( $P_{\text {rot }}=4.47$ days). (b): Time-variation of the rotational phase of the local photometric minima in the light curve of KIC 8482482. (c) and (d): Same as panels (a) and (d), but for KIC 10081606 ( $P_{\text {rot }}=7.46$ days). (e) and (f): Same as panels (a) and (d), but for KIC 10524994 ( $P_{\text {rot }}=11.9$ days). (g) and (h): Same as panels (a) and (d), but for KIC $11972298\left(P_{\text {rot }}=15.6\right.$ days). 
groups. We applied the same analysis to the light curves of the stars showing low amplitude rotational modulations like the Sun. Figure 13 represents the light curves and phase-time plots of KIC 5125098 $\left(P_{\text {rot }}=12.3\right.$ days), KIC 6801575 (11.9 days), KIC 3730378 (15.0 days), and KIC 9160630 (22.0 days). The area of starspots on these stars is estimated to be $\sim 2 \times 10^{-3} A_{1 / 2} \odot$ from the equation (3). We could not found any series of local minima which persist for $>100$ days from the phase-time plots of these stars. The majority of series of local brightness minima disappear within 3-4 stellar rotations, $\sim 50$ days, which is comparable to the lifetime of sunspots with the area of $\sim 5 \times 10^{-4} A_{1 / 2 \odot}(\sim 30$ days; Petrovay \& van Driel-Gesztelyi 1997). These results suggest that it is possible to extend the size-lifetime relation of sunspots by Petrovay \& van Driel-Gesztelyi (1997) up to the larger starspots with the area of $\sim 10^{-2} A_{1 / 2 \odot}$.

\section{Appendix 2 Fraction of flare stars}

As described in section 2, we used Shibayama et al. (2013) as the catalog of superflare stars. Recently, Davenport (2016) published the list of flare stars in the Kepler field. Figure 14 shows the fraction of flare stars with $5600 \leq T_{\text {eff }}<6300 \mathrm{~K}$ as a function of the rotation period derived from the list of flare stars by Shibayama et al. (2013) and that by Davenport (2016). In the short-period regime $\left(P_{\text {rot }}<3\right)$, the fraction of flare stars derived from Davenport (2016) is comparable to that from Shibayama et al. (2013). On the other hand, in the period range of $P_{\text {rot }}>3$ days, the fraction of flare stars derived from Davenport (2016) is significantly lower than that from Shibayama et al. (2013). Davenport (2016) selected only flare stars showing $>100$ flares during the Kepler observations (flare rate of $>26$ events per year). This difference can be explained by the lack of moderately active superflare stars in the flare star list by Davenport (2016). There are only a few flare stars with $P_{\text {rot }}>3$ days in the list by Davenport (2016). This suggests that the frequency of flares on the flare stars with $P_{\text {rot }}>3$ days are lower than that on the flare stars with $P_{\text {rot }}<3$ days.

\section{References}

Barnes, S. A. 2007, ApJ, 669, 1167

Bell, B., \& Glazer, H. 1959, Smithsonian Contributions to Astrophysics, 3, 25

Benz, A. O. 2008, Living Reviews in Solar Physics, 5, 1

Berdyugina, S. V. 2005, Living Reviews in Solar Physics, 2,8

Bogdan, T. J., Gilman, P. A., Lerche, I., \& Howard, R. 1988, ApJ, 327, 451

Boro Saikia, S., et al. 2016, A\&A, 594, A29 

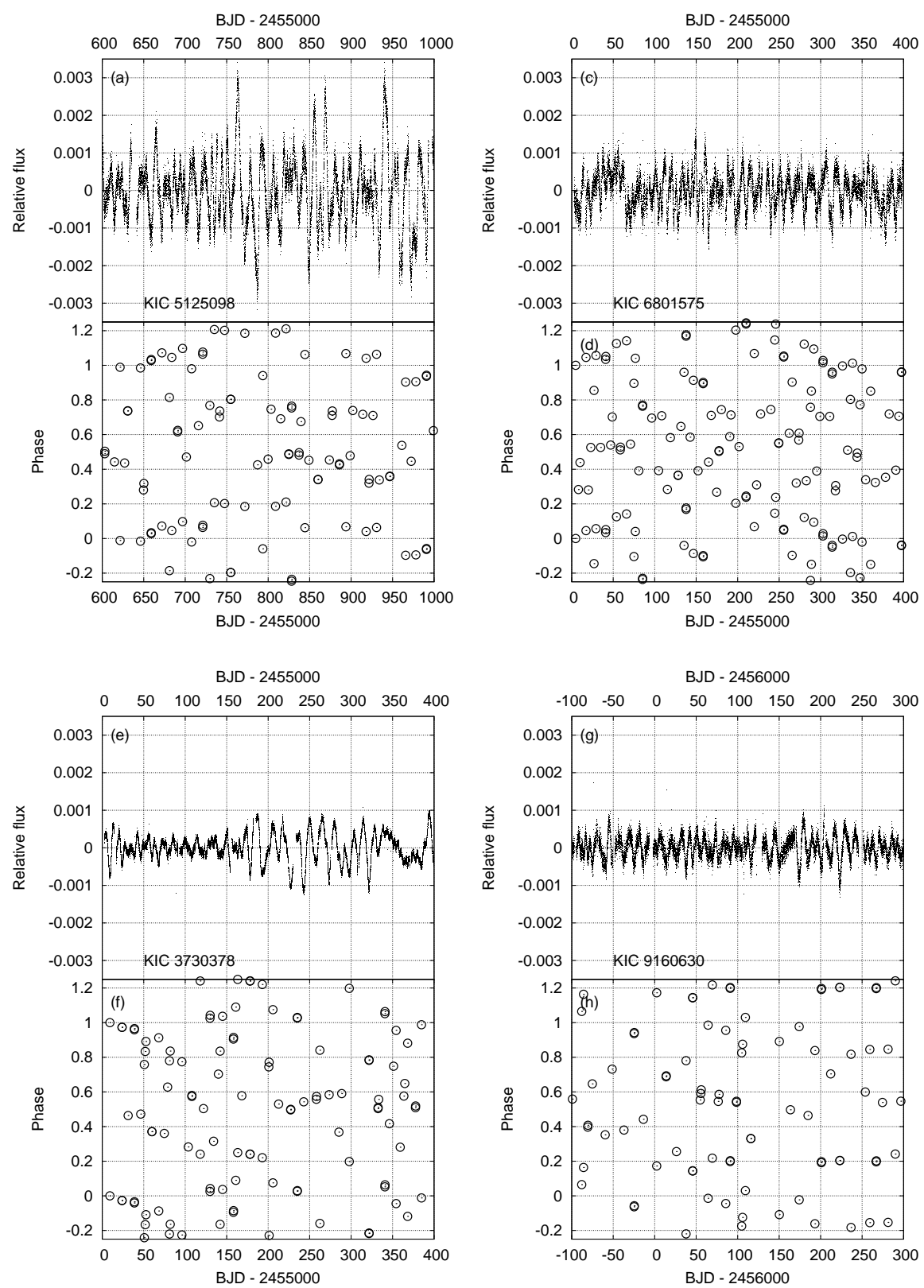

Fig. 13. (a): Light curve of the solar-type star KIC 5125098 ( $P_{\text {rot }}=12.3$ days). (b): Time-variation of the rotational phase of the local photometric minima in the light curve of KIC 5125098. (c) and (d): Same as panels (a) and (d), but for KIC 6801575 ( $P_{\text {rot }}=11.9$ days). (e) and (f): Same as panels (a) and (d), but for KIC 3730378 ( $P_{\text {rot }}=15.0$ days). $(\mathrm{g})$ and $(\mathrm{h})$ : Same as panels (a) and (d), but for KIC $9160630\left(P_{\text {rot }}=22.0\right.$ days). 


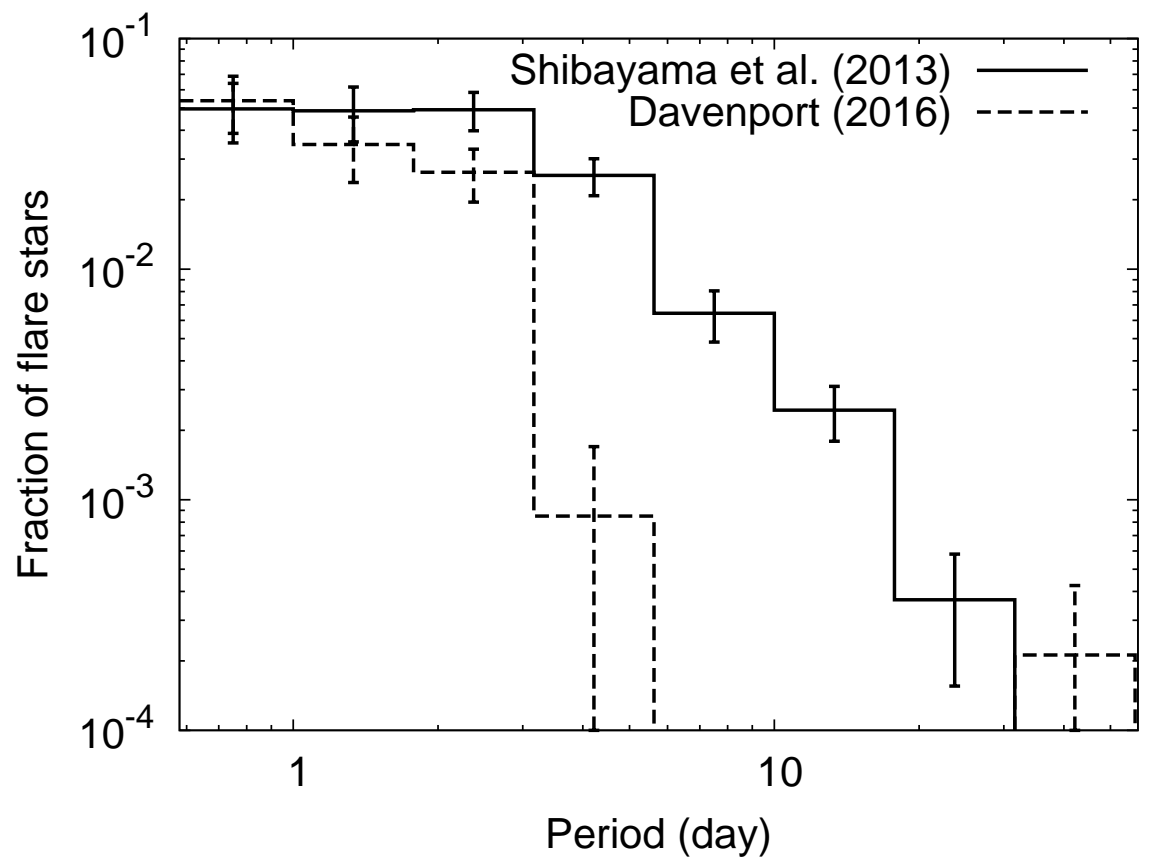

Fig. 14. The fraction of flare stars as a function of the rotation period. Solid line and dotted line represent the fraction of flare stars derived from Shibayama et al. (2013) and that from Davenport (2016). Since Davenport (2016) selected only flare stars showing $>100$ flares during the Kepler observations, only the extremely active stars would be listed in the list.

Brown, T. M., Latham, D. W., Everett, M. E., \& Esquerdo, G. A. 2011, AJ, 142, 112

Candelaresi, S., Hillier, A., Maehara, H., Brandenburg, A., \& Shibata, K. 2014, ApJ, 792, 67

Davenport, J. R. A. 2016, ApJ, 829, 23

Donati, J.-F., \& Landstreet, J. D. 2009, ARA\&A, 47, 333

Emslie, A. G., et al. 2012, ApJ, 759, 71

Fröhlich, H.-E., Frasca, A., Catanzaro, G., Bonanno, A., Corsaro, E., Molenda-akowicz, J., Klutsch, A., \& Montes, D. 2012, A\&A, 543, A146

Huber, D., et al. 2014, ApJS, 211, 2

Karoff, C., et al. 2016, Nature Communications, 7, 11058

Kippenhahn, R., \& Weigert, A. 1990, Stellar Structure and Evolution, Springer-Verlag Berlin Heidelberg New York

Kopp, G., Lawrence, G., \& Rottman, G. 2005, Sol. Phys., 230, 129

Maehara, H., et al. 2012, Nature, 485, 478

Maehara, H., Shibayama, T., Notsu, Y., Notsu, S., Honda, S., Nogami, D., \& Shibata, K. 2015, Earth, Planets, and Space, 67, 59

Mamajek, E. E., \& Hillenbrand, L. A. 2008, ApJ, 687, 1264-1293

Meibom, S., Mathieu, R. D., \& Stassun, K. G. 2009, ApJ, 695, 679 
Meibom, S., Barnes, S. A., Platais, I., Gilliland, R. L., Latham, D. W., \& Mathieu, R. D. 2015, Nature, 517, 589

McQuillan, A., Mazeh, T., \& Aigrain, S. 2014, ApJS, 211, 24

Morin, J., et al. 2008, MNRAS, 390, 567

Notsu, Y., et al. 2013, ApJ, 771, 127

Notsu, Y., Honda, S., Maehara, H., Notsu, S., Shibayama, T., Nogami, D., \& Shibata, K. 2015a, PASJ, 67, 32

Notsu, Y., Honda, S., Maehara, H., Notsu, S., Shibayama, T., Nogami, D., \& Shibata, K. 2015b, PASJ, 67, 33

Petrovay, K., \& van Driel-Gesztelyi, L. 1997, Sol. Phys., 176, 249

Pont, F., et al. 2007, A\&A, 476, 1347

Rauer, H., et al. 2014, Experimental Astronomy, 38, 249

Rice, J. B. 2002, Astronomische Nachrichten, 323, 220

Ricker, G. R., et al. 2015, Journal of Astronomical Telescopes, Instruments, and Systems, 1, 014003

Sammis, I., Tang, F., \& Zirin, H. 2000, ApJ, 540, 583

Schaefer, B. E., King, J. R., \& Deliyannis, C. P. 2000, ApJ, 529, 1026

Schrijver, C. J., et al. 2012, Journal of Geophysical Research (Space Physics), 117, A08103

Shibayama, T., et al. 2013, ApJS, 209, 5

Shibata, K., \& Magara, T. 2011, Living Reviews in Solar Physics, 8, 6

Shibata, K., et al. 2013, PASJ, 65, 49

Silva, A. V. R. 2003, ApJL, 585, L147

Strassmeier, K. G., \& Rice, J. B. 1998, A\&A, 330, 685

Weaver, W. B., \& Naftilan, S. A. 1973, PASP, 85, 213 